\title{
NLIE of Dirichlet sine-Gordon Model for Boundary Bound States
}

\author{
Changrim Ahn ${ }^{1}$, Zoltán Bajnok ${ }^{2}$, László Palla ${ }^{3}$, Francesco Ravanini ${ }^{4}$
}

November 24, 2018

\author{
${ }^{1}$ Department of Physics, Ewha Womans Univ., Seoul 120-750, South Korea \\ ${ }^{2}$ HAS Theoretical Physics Research Group, ${ }^{3}$ Department of Theoretical Physics, Roland Eötvös Univ. \\ H-1117 Budapest Pázmány s. 1/A, Hungary \\ ${ }^{4}$ Univ. of Bologna, Physics Department, INFN Section, Via Irnerio 46, Bologna 40126, Italy
}

We dedicate this work to the memory of Alyosha Zamolodchikov

\begin{abstract}
We investigate boundary bound states of sine-Gordon model on the finite-size strip with Dirichlet boundary conditions. For the purpose we derive the nonlinear integral equation (NLIE) for the boundary excited states from the Bethe ansatz equation of the inhomogeneous XXZ spin $1 / 2$ chain with boundary imaginary roots discovered by Saleur and Skorik. Taking a large volume (IR) limit we calculate boundary energies, boundary reflection factors and boundary Lüscher corrections and compare with the excited boundary states of the Dirichlet sine-Gordon model first considered by Dorey and Mattsson. We also consider the short distance limit and relate the IR scattering data with that of the UV conformal field theory.
\end{abstract}

\section{Introduction}

Boundary integrable field theories in two dimensions have been investigated mainly by two approaches. The boundary bootstrap approach determines the reflection amplitudes in a factorized $S$-matrix framework which is valid in the large volume (IR) limit [1]. In a small volume, on the other hand, the underlying quantum field theories can be described as perturbed boundary conformal field theories. Complete understanding is possible only after the two approaches are linked in such a way that the states and operators in the alternative formulations are exactly matched.

The nonlinear integral equation (NLIE) has been most effective in linking the two descriptions for the sine-Gordon model. The NLIE for the bulk theory has been started several years ago by various authors [2, 3]. An evident advantage of this method is that it can deal with excited states relatively easily as shown with great success in the bulk sine-Gordon model [4, 5]. The NLIE is a sort of continuum limit of the Bethe Ansatz equation (BAE) of an inhomogeneous alternating spin $1 / 2$ XXZ chain model which regularizes the sine-Gordon model while keeping integrability. This method has been extended to the sine-Gordon model defined on a strip with two boundaries. The ground state NLIE for the Dirichlet boundary conditions (BCs) has been studied in [6] and for general non-diagonal BCs in [7]. The bulk excited state NLIE for the Dirichlet BCs has been analyzed in [8] and the hole excited state for a general non-diagonal BCs in [9]. In the present paper we investigate how this method can be extended to the boundary excited states for Dirichlet BCs.

The complete spectrum of boundary excited states of the Dirichlet sine-Gordon (DSG) model on a half line with one boundary has been constructed by Dorey and Mattsson (DM) by inspecting the analytic structure of the reflection matrix in a bootstrap approach [10. They found a rich structure of excited boundary states, the boundary bound states (BBS). These states are the scattering states which can be defined only in the IR limit of the finite size setting. It is important to relate the IR states with the UV conformal states appearing in the small volume description for the complete understanding of 
the DSG model. In this paper we analyze carefully the "imaginary roots" of the boundary XXZ BAE, first discovered by Saleur and Skorik [11. From this we derive the NLIE including the imaginary roots which describes the BBSs of the DSG in the whole scale. Taking the IR limit of the NLIE, we can show that there is a one to one correspondence between the purely imaginary roots and the DM BBSs.

The paper is organized as follows: In section 2 we summarize the available results of the DSG model. We start by analyzing the conditions for the existence of the imaginary roots of the inhomogeneous spin $1 / 2$ XXZ model. The NLIE, derived from the lattice BAE, contains parameters originating from the XXZ model which should be compared with the bootstrap solution [10]. We analyze the large volume limit of the NLIE in section 3 and find agreement with the BAE classification. This leads to full physical interpretation of the energy levels described by the NLIE without any source and the one with imaginary roots only. We do it in two steps, first with the simpler repulsive case and then the more complicated attractive one. In both cases the proposed correspondence between the various source terms and the DM spectrum of BBSs are derived from the soliton and breather reflection amplitudes and matching of the boundary energies. As a final check we compare the finite size energy correction derived from the NLIE to the boundary Lüscher correction [12]. Section 4 deals with the calculation of the conformal dimensions of underlying boundary conformal field theory by taking the UV limit of the NLIE, which gives another convincing support of our result. We conclude and give the outlook for future investigations in section 5 .

\section{Derivation of the NLIE}

In this section we summarize the results available in the literature tailor-made for future applications.

\subsection{Imaginary Roots of the Bethe Ansatz equation}

To derive the NLIE for the DSG model, we consider anti-ferromagnetic XXZ spin $1 / 2$ model in a chain of $N$ sites with lattice spacing $a$, coupled to parallel magnetic fields $h_{-}$and $h_{+}$at the left and right boundaries, respectively. Its Hamiltonian is written as

$$
\mathcal{H}\left(\gamma, h_{+}, h_{-}\right)=-J \sum_{n=1}^{N-1}\left(\sigma_{n}^{x} \sigma_{n+1}^{x}+\sigma_{n}^{y} \sigma_{n+1}^{y}+\cos \gamma \sigma_{n}^{z} \sigma_{n+1}^{z}\right)+h_{+} \sigma_{1}^{z}+h_{-} \sigma_{N}^{z} .
$$

Here $\sigma_{n}^{\alpha}, \alpha=x, y, z$ are Pauli matrices on the $n$-th site and the anisotropy is $0 \leq \gamma \pi$. Whenever necessary, we will use another coupling constant $p$ defined by

$$
p=\frac{\pi}{\gamma}-1, \quad 0<p<\infty .
$$

The BAEs for the boundary XXZ chain (11) have been derived by Alcaraz et al. [13] and Sklyanin 14] using an algebraic Bethe ansatz approach. The BAEs are coupled equations for a set of $M$ roots which have distinct values $\theta_{1}, \ldots, \theta_{M}$ with $M \leq N / 2$;

$$
\left[s_{1}\left(\theta_{j}+\Lambda\right) s_{1}\left(\theta_{j}-\Lambda\right)\right]^{N} s_{H_{+}}\left(\theta_{j}\right) s_{H_{-}}\left(\theta_{j}\right)=\prod_{k=1, k \neq j}^{M} s_{2}\left(\theta_{j}-\theta_{k}\right) s_{2}\left(\theta_{j}+\theta_{k}\right)
$$

where we introduced a short notation

$$
s_{\nu}(x)=\frac{\sinh \frac{\gamma}{\pi}\left(x+\frac{i \nu \pi}{2}\right)}{\sinh \frac{\gamma}{\pi}\left(x-\frac{i \nu \pi}{2}\right)} .
$$

The boundary parameters $H_{ \pm}$in the BAEs are related to those in the Hamiltonian by

$$
h_{ \pm}=\sin \frac{\pi}{p+1} \cot \frac{2 \pi\left(H_{ \pm}+1\right)}{p+1} .
$$


In addition to real and complex roots, we are interested in the "imaginary" roots which have vanishing real parts. These objects, first observed by Saleur and Skorik [11], depend on the boundary parameters of both sides independently. For simplicity, we set the value of $h_{-}$so that it does not introduce any imaginary root and recall from [11] how the existence and locations of the imaginary roots depend on the values of $h_{+}$in the limits when $\Lambda \rightarrow \infty$ and $N \rightarrow \infty$. Defining $\kappa_{j}$ by

$$
e^{\kappa_{j}}=s_{1}\left(\theta_{j}+\Lambda\right) s_{1}\left(\theta_{j}-\Lambda\right),
$$

one can see that an imaginary root with $\theta_{j}=i u_{j}$ in the $\Lambda \rightarrow \infty$ limit satisfies

$$
\kappa_{j}=2 A \sin \gamma \sin \frac{2 \gamma u_{j}}{\pi}+O\left(e^{\frac{-4 \gamma \Lambda}{\pi}}\right), \quad \text { with } \quad A=\left[\cosh \frac{2 \gamma \Lambda}{\pi}\right]^{-1} .
$$

Now we look for some "string" solution in the form of

$$
\theta_{j}=i\left(-\frac{\pi H_{+}}{2}+j p \pi+\epsilon_{j}\right)
$$

where $\epsilon_{j}$ 's are supposed to be exponentially small in the $N \rightarrow \infty$ limit. Following [11], we denote it as " $(n, m)$ string" if $j$ can take integer values from $-n$ to $m$. Then, the BAEs take the following form

$$
\begin{aligned}
& e^{\kappa_{m} N} \propto \epsilon_{m}-\epsilon_{m-1} ; \quad e^{\kappa_{m-1} N} \propto \frac{\epsilon_{m-1}-\epsilon_{m-2}}{\epsilon_{m}-\epsilon_{m-1}} ; \ldots ; e^{\kappa_{l} N} \propto \frac{\epsilon_{l}-\epsilon_{l-1}}{\epsilon_{l+1}-\epsilon_{l}} ; \ldots ; e^{\kappa_{0} N} \epsilon_{0} \propto \frac{\epsilon_{0}-\epsilon_{-1}}{\epsilon_{1}-\epsilon_{0}} \\
& e^{\kappa_{-1} N} \propto \frac{\epsilon_{-1}-\epsilon_{-2}}{\epsilon_{0}-\epsilon_{-1}} ; \ldots ; e^{\kappa_{-n+1} N} \propto \frac{\epsilon_{-n+1}-\epsilon_{-n}}{\epsilon_{-n+2}-\epsilon_{-n+1}} ; \quad e^{\kappa_{-n} N} \propto \frac{1}{\epsilon_{-n+1}-\epsilon_{-n}}
\end{aligned}
$$

where we have omitted finite factors in the proportionality. Since all the $\epsilon_{j}$ are small we can determine them recursively starting from both $-n$ and $m$. They are consistent provided

$$
\begin{aligned}
& \kappa_{m}<0 \quad, \quad \kappa_{m}+\kappa_{m-1}<0 \quad, \ldots, \quad \kappa_{m}+\kappa_{m-1}+\ldots+\kappa_{1}<0, \\
& \kappa_{-n}>0 \quad, \quad \kappa_{-n}+\kappa_{-n+1}>0 \quad, \ldots, \kappa_{-n}+\kappa_{-n+1}+\ldots+\kappa_{-1}>0,
\end{aligned}
$$

and

$$
\kappa_{-n}+\kappa_{-n+1}+\ldots+\kappa_{-1}+\kappa_{0}+\kappa_{m}+\kappa_{m-1}+\ldots+\kappa_{1}>0 .
$$

In solving these inequalities we are interested in the domain in $H_{+}$for a fixed $p$ in which the $(n, m)$ string can exists. Since at some point we want to make connection with the BBSs we introduce the parameter:

$$
H_{+}=p\left(1-2 \xi_{+} / \pi\right)
$$

For small/large enough $\xi_{+}$the boundary state is absent and we are going to determine the value of $\xi_{+}$at which such a string can enter/leave the physical strip. For this we plot $\sin \left(\frac{2 \gamma}{\pi} u_{j}\right)$ which is the relevant part of $\kappa_{j}$ together with the $(n, m)$ string on Figure 1.

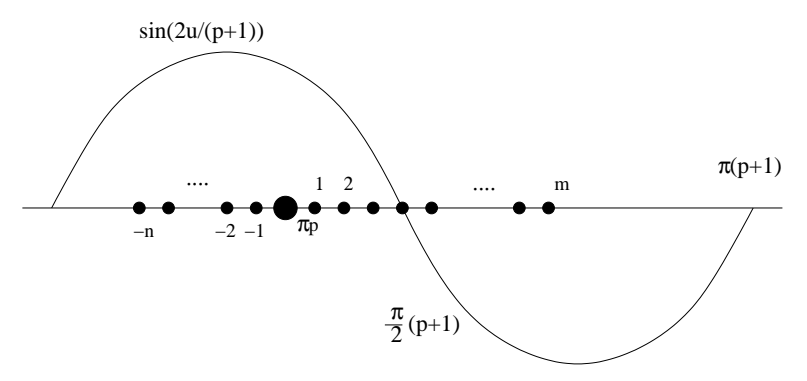

Figure 1: The boundary condition dependent factor of $\kappa_{j}$ in the $(n, m)$ string 
From (2) we can see that such a state appears when the average of $u_{1}$ and $u_{m}$ is exactly

$$
p \xi_{0}=\frac{\pi}{2}(1+p) .
$$

So for the existence we need $u_{1}+u_{m}>\pi(p+1)$ that is

$$
\xi_{+}>\frac{\pi}{2 p}-(m-1) \frac{\pi}{2}
$$

From (3) we can see that $u_{-n}>0$ is also needed that is

$$
\xi_{+} \geq(2 n+1) \frac{\pi}{2}
$$

Finally from (3) we can see that the state disappears when $u_{-n}+u_{m}>\pi(p+1)$ since we have too many points in the negative part of the sine function. So for the existence of the $(n, m)$ string we also need

$$
\xi_{+}<\frac{\pi}{2 p}+(n+2-m) \frac{\pi}{2} .
$$

\subsection{NLIE: the continuum limit of the BAE}

Based on the BAE, the NLIE equation determining the counting function $Z(\theta)$ in the continuum limit $N \rightarrow \infty$ can be written as 8

$$
\begin{aligned}
Z(\theta) & =2 M L \sinh \theta+g\left(\theta \mid\left\{\theta_{k}\right\}\right)+P_{\mathrm{bdry}}(\theta) \\
& -2 i \operatorname{Im} \int d x G(\theta-x-i \epsilon) \log \left[1-(-1)^{M_{S C}} e^{i Z(x+i \epsilon)}\right]
\end{aligned}
$$

where $P_{\mathrm{bdry}}(\theta)$ is the boundary contribution given by

$$
\begin{aligned}
& P_{\text {bdry }}(\theta)=2 \pi \int_{0}^{\theta} d x\left[F\left(x, H_{+}\right)+F\left(x, H_{-}\right)+G(x)+J(x)\right] \\
& G(\theta)=\int_{-\infty}^{\infty} \frac{d k}{2 \pi} \frac{\sinh \frac{\pi}{2}(p-1) k}{2 \sinh \frac{\pi}{2} p k \cosh \frac{\pi}{2} k} e^{i k \theta}, \quad \text { for } \quad|\operatorname{Im} \theta|<\pi \min (1, p) \\
& J(\theta)=\int_{-\infty}^{\infty} \frac{d k}{2 \pi} \frac{\sinh \frac{\pi}{4}(p-1) k \cosh \frac{\pi}{4}(p+1) k}{\sinh \frac{\pi}{2} p k \cosh \frac{\pi}{2} k} e^{i k \theta}, \quad \text { for } \quad|\operatorname{Im} \theta|<\frac{\pi}{2} \min (1, p) \\
& F(\theta, H)=\int_{-\infty}^{\infty} \frac{d k}{2 \pi} \operatorname{sign}(H) \frac{\sinh \frac{\pi}{2}(p+1-|H|) k}{2 \sinh \frac{\pi}{2} p k \cosh \frac{\pi}{2} k} e^{i k \theta}, \quad \text { for } \quad|\operatorname{Im} \theta|<\frac{\pi}{2}|H| .
\end{aligned}
$$

We have introduced a mass scale $M$ which will be identified with that of a soliton and the finite size $L$ by

$$
L=N a, \quad M=\frac{2}{a} e^{-\Lambda} .
$$

The "source" term is given by

$$
g\left(\theta \mid\left\{\theta_{k}\right\}\right)=\sum_{k} c_{k}\left[\chi_{(k)}\left(\theta-\theta_{k}\right)+\chi_{(k)}\left(\theta+\theta_{k}\right)\right]
$$

where

$$
\chi(\theta)=2 \pi \int_{0}^{\theta} d x G(x)
$$

and $\left\{\theta_{k}\right\}$ is the set of position of the various objects (holes, close and wide roots, specials) characterizing a certain state. They satisfy the quantization rule

$$
Z\left(\theta_{j}\right)=2 \pi I_{j} \quad, \quad I_{j} \in \mathbb{Z}+\frac{\rho}{2} \quad \rho=M_{S C} \bmod 2 .
$$


The coefficients $c_{k}$ are given by

$$
c_{k}= \begin{cases}+1 & \text { for holes } \\ -1 & \text { for all other objects }\end{cases}
$$

and for any function $f(\vartheta)$ we define

$$
f_{(k)}(\theta)= \begin{cases}f_{\mathrm{II}}(\theta) & \text { for wide roots } \\ f(\theta+i \epsilon)+f(\theta-i \epsilon) & \text { for specials } \\ f(\theta) & \text { for all other objects }\end{cases}
$$

where the second determination of $f(\theta)$ is defined by

$$
f_{\mathrm{II}}(\theta)=\left\{\begin{array}{ccc}
f(\theta)+f(\theta-i \pi \operatorname{sign} \operatorname{Im} \theta) & \text { if } & p>1 \\
f(\theta)-f(\theta-i \pi p \operatorname{sign} \operatorname{Im} \theta) & \text { if } \quad p<1
\end{array} \text { for }|\operatorname{Im} \theta|>\pi \min (1, p)\right.
$$

For the vacuum state containing real roots only, Eq.(4) coincides with the one found some years ago in [6]. Once the equation is solved for $Z(\theta+i \epsilon)$ one can use this result to compute the $Z(\theta)$ function at any value in the analyticity strip $|\operatorname{Im} \theta|<\pi \min (1, p)$, provided the function $P_{\text {bdry }}(\theta)$ is well defined there. To extend the function outside this analyticity strip one has to resort to the following modification of the NLIE

$$
\begin{aligned}
Z(\theta) & =2 M L \sinh _{\mathrm{II}} \theta+g_{\mathrm{II}}\left(\theta \mid\left\{\theta_{k}\right\}\right)+P_{\mathrm{bdry}}(\theta) \\
& -2 i \operatorname{Im} \int d x G_{\mathrm{II}}(\theta-x-i \epsilon) \log \left[1-(-1)^{M_{S C}} e^{i Z(x+i \epsilon)}\right] .
\end{aligned}
$$

The continuum limit of the counting equation which restrict the allowed root configuration is given by

$$
N_{H}-2 N_{S}=\frac{1}{2}\left(\operatorname{sign}\left(H_{+}\right)+\operatorname{sign}\left(H_{+}\right)\right)-1+M_{C}+2 M_{W} \operatorname{step}(p-1)+m .
$$

(The integer $m$ appearing here is related to possible winding of the sine-Gordon field, see next section).

Once $Z(\theta)$ is known, it can be used to compute the energy. It is composed of bulk and boundary terms whose expression can be found in [6] and a Casimir energy scaling function given by

$$
E=M \sum_{k} c_{k} \cosh _{(k)} \theta_{k}-M \int \frac{d x}{4 \pi} \sinh x Q(x) . \quad ; \quad Q(x)=2 \Im m \log \left[1-(-1)^{M_{S C}} e^{i Z(x+i \epsilon)}\right]
$$

\subsection{Relation to Boundary sine-Gordon model}

The continuum limit of the inhomogeneous XXZ spin $1 / 2$ chain describes the sine-Gordon model. If we introduce diagonal ( $\sigma_{z}$ only) boundary condition on the spin chain, the continuum limit should describe the DSG model, whose action can be written as

$$
\mathcal{A}_{D S G}=\frac{1}{2} \int_{-\infty}^{\infty} d t \int_{0}^{L} d x\left[\left(\partial_{\mu} \phi\right)^{2}+\frac{2 m_{0}^{2}}{\beta^{2}} \cos \beta \phi\right]
$$

with the Dirichlet boundary conditions

$$
\phi(0, t) \equiv \phi_{-}+\frac{2 \pi}{\beta} m_{-} \quad ; \quad \phi(L, t)=\phi_{+}+\frac{2 \pi}{\beta} m_{+}, \quad m_{ \pm} \in \mathbb{Z} .
$$

Notice that the bulk and boundary parameters of the DSG and spin chain models are related by [6]

$$
p^{-1}=\frac{8 \pi}{\beta^{2}}-1, \quad ; \quad H_{ \pm}=p\left(1 \mp \frac{8}{\beta} \phi_{ \pm}\right) .
$$


This model has several important applications ranging from condensed matter physics to string theory. An important feature of the DSG model is the conservation of the topological charge

$$
Q \equiv \frac{\beta}{2 \pi}\left[\int_{0}^{L} d x \frac{\partial}{\partial x} \phi(x, t)-\phi_{+}+\phi_{-}\right]=m_{+}-m_{-} \in \mathbb{Z}
$$

The model enjoys the discrete symmetry of the field $\phi \rightarrow \phi+\frac{2 \pi}{\beta} m$ and simultaneously $\phi_{ \pm} \rightarrow \phi_{ \pm}+$ $\frac{2 \pi}{\beta} m(m \in \mathbb{Z})$. The charge conjugation symmetry $\phi \rightarrow-\phi$ sending solitons into anti-solitons is also guaranteed, provided $\phi_{ \pm} \rightarrow-\phi_{ \pm}$simultaneously.

The well known bulk particle spectrum of sine-Gordon model is composed of solitons and antisolitons with topological charge 1 and -1 respectively, and their bound states known as breathers in the attractive regime $0<\beta \leq \sqrt{4 \pi}$ and of course they are also part of the DSG spectrum. Another important part of the spectrum of the DSG model in the half line theory - i.e. in the presence of one boundary only - is the complicated spectrum of the BBSs described in [10]. In addition to the bulk S-matrices [15] and boundary reflection matrices for the soliton or anti-solitons [1, the complete excited boundary reflection matrices in the presence of the BBSs have been found in [10].

The DM result on the BBSs of the half line theory can be summarized as follows. First, define two sets of variables

$$
\begin{aligned}
\nu_{n} & =\xi p-\frac{(2 n+1) \pi p}{2}=\nu_{0}-n p \pi \quad ; \quad n \geq 0 \\
w_{m} & =\pi-\xi p-\frac{(2 m-1) \pi p}{2}=\pi-\nu_{-m} .
\end{aligned}
$$

where the bootstrap parameters $p$ and $\xi$ are related to those of the Lagrangian as

$$
\frac{p+1}{p}=\frac{8 \pi}{\beta^{2}} \quad ; \quad \xi_{ \pm}=\frac{4 \pi}{\beta} \phi_{ \pm}
$$

Whenever a condition

$$
\frac{\pi}{2}>\nu_{n_{1}}>w_{m_{2}}>\nu_{n_{2}}>\ldots>w_{m_{k}}>\nu_{n_{k}}>\ldots>0
$$

is satisfied, a BBS can exist. If the last variable is of $\nu$ type, then the BBS is denoted as

$$
\left|1 ; n_{1}, m_{1}, \ldots, n_{k-1}, m_{k-1}, n_{k}\right\rangle
$$

and if it is of $w$ type, then the state is denoted as

$$
\left|0 ; n_{1}, m_{1}, \ldots, n_{k-1}, m_{k-1}, n_{k}, m_{k}\right\rangle .
$$

The energy of such a state relative to the ground state is given by

$$
E_{\left|0 / 1 ; n_{1}, m_{1}, \ldots, n_{k-1}, m_{k-1}, \ldots\right\rangle}=\sum_{j} M \cos \nu_{n_{j}}+\sum_{j} M \cos w_{m_{j}} .
$$

The boundary reflection matrix on excited boundaries can also be derived. We denote by $P_{|0\rangle}^{+}(\xi, \theta)$ the boundary reflection matrix element of a soliton on the ground state boundary. The boundary parameter dependent part reads as 11]

$$
-i \frac{d}{d \theta} \log \frac{P_{|0\rangle}^{+}(\theta, \xi)}{R_{0}(\theta)}=\int_{-\infty}^{\infty} d k \mathrm{e}^{i k \theta} \frac{\sinh \left(\frac{\pi}{2}\left(1+\frac{2 \xi p}{\pi}\right) k\right)}{2 \sinh \frac{\pi}{2} p k \cosh \frac{\pi}{2} k}
$$

The reflection factor of solitons on the $|1 ; 0\rangle$ excited boundary is given by

$$
P_{|1 ; 0\rangle}^{+}(\theta, \xi)=P_{|0\rangle}^{+}(\theta, \xi) a\left(\theta-i \nu_{0}\right) a\left(\theta+i \nu_{0}\right) \equiv P_{|1\rangle}^{+}(\theta, \xi)
$$


where $a(\theta)=e^{i \chi(\theta)}$ describes the soliton-soliton S-matrix element. The reflection factor on the general boundary is given by

$$
P_{\left|0 / 1 ; n_{1}, m_{1}, \ldots, n_{k-1}, m_{k-1}, \ldots\right\rangle}^{+}=P_{|0 / 1\rangle}^{+} \prod_{k} \frac{a\left(\theta-i \nu_{n_{k}}\right) a\left(\theta+i \nu_{n_{k}}\right)}{a\left(\theta-i \nu_{0}\right) a\left(\theta+i \nu_{0}\right)} \prod_{k} \frac{a\left(\theta-i w_{m_{k}}\right) a\left(\theta+i w_{m_{k}}\right)}{a\left(\theta-i w_{0}\right) a\left(\theta+i w_{0}\right)} .
$$

Using the identity

$$
\frac{a\left(\theta-i w_{m_{k}}\right) a\left(\theta+i w_{m_{k}}\right) a\left(\theta-i\left(\pi-w_{m_{k}}\right)\right) a\left(\theta+i\left(\pi-w_{m_{k}}\right)\right)}{a\left(\theta-i \nu_{0}\right) a\left(\theta+i \nu_{0}\right) a\left(\theta-i w_{0}\right) a\left(\theta+i w_{0}\right)}=1 .
$$

which comes from the unitarity and crossing symmetry of the bulk S-matrix, the general reflection factor is equivalent to

$$
P_{\left|0 / 1 ; n_{1}, m_{1}, \ldots, n_{k-1}, m_{k-1}, \ldots\right\rangle}^{+}=P_{|0 / 1\rangle}^{+} \prod_{k} \frac{a\left(\theta-i \nu_{n_{k}}\right) a\left(\theta+i \nu_{n_{k}}\right)}{a\left(\theta-i\left(\pi-w_{m_{k}}\right)\right) a\left(\theta+i\left(\pi-w_{m_{k}}\right)\right)} .
$$

On physical grounds one expects that the DSG model on the strip with two boundaries should have in general pairs of the DM type BBS in the spectrum when $L \rightarrow \infty$. In this paper we consider a somewhat simpler situation where the boundary parameters on one boundary do not allow any DM bound state. In this case, one expects that only one set of the DM BBSs are present in $L \rightarrow \infty$ limit. In the following we show how the solutions of the NLIE with purely imaginary roots meet this expectation.

\section{Large volume behavior of the NLIE}

In this section we provide the interpretation of the boundary strings of the NLIE in the large volume limit by mapping them to the boundary bound states classified by DM. First we show that the asymptotic analysis for the existence of a boundary string in NLIE is equivalent to the BAE analysis, then we focus on their interpretation. For pedagogical reasons we present the results for the repulsive regime first, where we have at most one BBS and then turn to the more complicated problem of the attractive regime. Finally we confirm our findings by calculating the boundary Lüscher corrections for the ground-states.

\subsection{Large volume analysis of the boundary excited state NLIE}

The aim of this subsection is to replace the $\epsilon$ analysis for the $(n, m)$ strings in the BAE by a source term analysis in the infrared (IR) limit of the NLIE (4). In this analysis the counting function $Z(\theta)$ is replaced by its asymptotic (large volume) form:

$$
Z(\theta)=2 M L \sinh \theta+P_{\mathrm{bdry}}(\theta)-\sum_{k}\left(\chi\left(\theta-\theta_{k}\right)+\chi\left(\theta+\theta_{k}\right)\right),
$$

and the quantization condition is obtained from

$$
e^{i Z\left(\theta_{j}\right)}=1 . \quad j=1, \ldots
$$

Using the relations (8) and (9) the boundary parameters $H_{ \pm}$are related to the DM $\xi_{ \pm}$parameters as

$$
H_{ \pm}=p\left(1 \mp \frac{2 \xi_{ \pm}}{\pi}\right)
$$

Some care is required for Eqs.(1415) since one may have to use $\chi_{\text {wide }}(\theta)$ instead of $\chi(\theta)$ depending on the location of the roots and also the second determination form of all the quantities. The expectation is that from this asymptotic analysis one can obtain the same string like objects as from the $\epsilon$ analysis 
in the BAE. This would confirm the relevance of the results of the $\epsilon$ analysis, as here we work with the (large volume limit of the) exact ground state(s) as opposed to the pseudo-vacuum in the $\epsilon$ analysis.

In this analysis we keep $H_{-}$in the domain where we expect no bound state on this boundary $\left(0<H_{-}<2 p\right)$ while we let $H_{+}$to move from a similar domain into $-1<H_{+}<0$ where bound states are expected. Consider first the zero string case when in Eq.(14) there are just two source terms with $\pm \theta_{0}$ and assume $H_{+}$is positive $0<H_{+}$(i.e. $\xi_{+}<\pi / 2$ ). Using the well known identity

$$
e^{i P_{\mathrm{bdry}}(\theta)}=-\frac{P_{|0\rangle}^{+}\left(\theta, \xi_{+}\right) P_{|0\rangle}^{+}\left(\theta, \xi_{-}\right)}{a(2 \theta)}
$$

where $P_{|0\rangle}^{+}(\theta, \xi)$ is the Ghoshal-Zamolodchikov ground state soliton reflection amplitude (11) and $a(\theta)$ denotes the bulk soliton-soliton scattering the only equation in Eq.(15) becomes:

$$
e^{i 2 M L \sinh \theta_{0}} \frac{P_{|0\rangle}^{+}\left(\theta_{0}, \xi_{+}\right) P_{|0\rangle}^{+}\left(\theta_{0}, \xi_{-}\right)}{a\left(2 \theta_{0}\right) a\left(2 \theta_{0}\right)}=1 .
$$

An imaginary root corresponding to a BBS would show up in the form of a solution $\theta_{0}=i v_{0}+\epsilon$ with $v_{0}$ in the physical domain $\left(0<v_{0}<\frac{\pi}{2}\right)$ and $\epsilon \rightarrow 0$ for $L \rightarrow \infty$. This can happen only if $i v_{0}$ is a pole of one of the $P^{+}$'s. However, they have no poles in the physical strip when both $\xi_{ \pm}<\frac{\pi}{2}$. (Note that the $a$ 's in the denominator cancel also the boundary independent poles of the two $P^{+}{ }^{\text {s }}$ ). Thus for both $H_{ \pm}$positive this asymptotic analysis gives no hint of a bound state. This is also consistent with the counting equation (6) that for $H_{ \pm}>0$ and $N_{H}=0$ allows only a solution with $M_{c}=0$.

Now let $H_{+}$become negative, but still consider the zero string case since the counting equation now allows $M_{c}=1$. The crucial observation is that the exponential of $i P_{\mathrm{bdry}}(\theta)$ contains in this case the excited state soliton reflection amplitude $P_{|1 ; 0\rangle}^{+}\left(\theta, \xi_{+}\right)$:

$$
\left.e^{i P_{\mathrm{bdry}}(\theta)}\right|_{-1<H_{+}<0}=-\frac{P_{|1 ; 0\rangle}^{+}\left(\theta, \xi_{+}\right) P_{|0\rangle}^{+}\left(\theta, \xi_{-}\right)}{a(2 \theta)} .
$$

In DM [10] $P_{|1 ; 0\rangle}^{+}\left(\theta, \xi_{+}\right)$is expressed in two equivalent ways:

$$
P_{|1 ; 0\rangle}^{+}\left(\theta, \xi_{+}\right)=P_{|0\rangle}^{+}\left(\theta, \xi_{+}\right) a\left(\theta-\nu_{0}\right) a\left(\theta+\nu_{0}\right), \quad P_{|1 ; 0\rangle}^{+}\left(\theta, \xi_{+}\right)=\overline{P_{|0\rangle}^{-}\left(\theta, \xi_{+}\right)}=P_{|0\rangle}^{+}\left(\theta, \xi_{+}-\frac{\pi}{p}-\pi\right) .
$$

The first form is natural from the bootstrap point of view and makes it easy to see that $P_{|1 ; 0\rangle}^{+}\left(\theta, \xi_{+}\right)$ has poles at $i \nu_{0}$ and at $i \nu_{-N}$ for $N=1,2, \ldots$, while the second form (where the over-line describes the transformation $\left.\xi_{+} \rightarrow \pi\left(1+p^{-1}\right)-\xi_{+}\right)$is useful to verify the integral representation. To support the claim we write here $F\left(\theta, H_{+}\right)$for $H_{+}>0$ :

$$
F\left(\theta, H_{+}\right)=\int_{-\infty}^{\infty} \frac{d k}{2 \pi} \mathrm{e}^{i k \theta} \frac{\sinh \left(k p\left(\xi_{+}+\frac{\pi}{2 p}\right)\right)}{2 \sinh \frac{p \pi}{2} k \cosh \frac{\pi}{2} k},
$$

and for $H_{+}<0$ :

$$
F\left(\theta, H_{+}\right)=\int_{-\infty}^{\infty} \frac{d k}{2 \pi} \mathrm{e}^{i k \theta} \frac{\sinh \left(k p\left(\xi_{+}-\pi-\frac{\pi}{2 p}\right)\right)}{2 \sinh \frac{p \pi}{2} k \cosh \frac{\pi}{2} k},
$$

showing that they are indeed connected by the transformation in the second DM form. Note that this implies that in this domain of $H_{ \pm}$the integral equation describes the excited state $|1 ; 0\rangle$.

Using this observation in Eq.(15) leads to the quantization condition

$$
e^{i 2 M L \sinh \theta_{0}} \frac{P_{|1 ; 0\rangle}^{+}\left(\theta_{0}, \xi_{+}\right) P_{|0\rangle}^{+}\left(\theta_{0}, \xi_{-}\right)}{a\left(2 \theta_{0}\right) a\left(2 \theta_{0}\right)}=1 .
$$

Since $P_{|1 ; 0\rangle}^{+}\left(\theta, \xi_{+}\right)$has poles in the physical strip this equation admits a bound state solution

$$
\theta_{0}=i\left(\nu_{0}+\epsilon\right) \quad \text { with } \quad \epsilon \sim R e^{-2 M L \sin \nu_{0}},
$$


(where $i R$ is the residue of the pole at $i \nu_{0}$ ) satisfying the requirements described earlier. Eq.(18) is correct if $\theta_{0}$ is in the first determination; but this condition is met in a domain where $\xi_{+}$just exceeds $\pi / 2\left(H_{+}\right.$is just below 0 ) both in the $p>1$ (repulsive) and in the $p<1$ (attractive) domains. Even for these $\xi_{+}$-S the poles of $P_{|1 ; 0\rangle}^{+}\left(\theta, \xi_{+}\right)$at $i \nu_{-N}$ are in the second determination thus cannot be used to find solutions to Eq.(18) since the form of the equation changes there. Furthermore in the repulsive regime also the counting equation would require to introduce something else (possibly moving objects) to compensate the presence of the wide roots.

If $\xi_{+}$exceeds $3 \pi / 2$ then also $i \nu_{0}$ gets into second determination $\left(\nu_{0}>p \pi\right)$ and we have to reconsider the asymptotic analysis and the solution we found even for the zero string case. (Since $\xi_{+} \leq \xi_{\max }=$ $\frac{\pi}{2}\left(1+p^{-1}\right), \xi_{+}=3 \pi / 2$ is in this allowed range only if $p<1 / 2$ in the attractive domain). In the quantization condition, Eq.(15), now $Z\left(\theta_{0}\right)_{I I}$ appears, where

$$
Z\left(\theta_{0}\right)_{I I}=2 M L\left(\sinh \theta_{0}-\sinh \left(\theta_{0}-i p \pi\right)\right)+P_{\mathrm{bdry}}\left(\theta_{0}\right)-P_{\mathrm{bdry}}\left(\theta_{0}-i p \pi\right)-\text { source }
$$

with

$$
\text { source }=\left(\chi\left(2 \theta_{0}\right)+\chi\left(2 \theta_{0}-2 i p \pi\right)-2 \chi\left(2 \theta_{0}-i p \pi\right)\right) .
$$

As a consequence Eq.(15) now takes the form:

$$
e^{i 2 M L\left(\sinh \theta_{0}-\sinh \left(\theta_{0}-i p \pi\right)\right)} \frac{P_{|1 ; 0\rangle}^{+}\left(\theta_{0}, \xi_{+}\right) P_{|0\rangle}^{+}\left(\theta_{0}, \xi_{-}\right)}{a\left(2 \theta_{0}\right) a\left(2 \theta_{0}\right)} \frac{\left(a\left(2 \theta_{0}-i p \pi\right)\right)^{2}}{P_{|1 ; 0\rangle}^{+}\left(\theta_{0}-i p \pi, \xi_{+}\right) P_{|0\rangle}^{+}\left(\theta_{0}-i p \pi, \xi_{-}\right)}=1 .
$$

Note that the $P_{|1 ; 0\rangle}^{+}$in the denominator cancels all but the $i \nu_{0}$ pole of $P_{|1 ; 0\rangle}^{+}$in the numerator, thus Eq.(19) admits only a bound state solution of the form

$$
\theta_{0}=i\left(\nu_{0}+\epsilon\right) \quad \text { with } \quad \epsilon \sim R e^{-2 M L\left(\sin \nu_{0}-\sin \left(\nu_{0}-p \pi\right)\right)} .
$$

Thus the asymptotic analysis gives a possibility for a zero string bound state solution if $H_{+}<0$ independently whether $i \nu_{0}$ is in the first or in the second determination. However, since the source terms are different in the two cases $\left(\chi \rightarrow \chi_{\text {wide }}\right.$ for $i \nu_{0}$ in the second determination), the interpretation of the bound states is different: while in the first case it corresponds to the ground state, in the second it corresponds to the state $|1 ; 1\rangle$ as described in subsection 3.2 .

Let's now turn to longer strings that may appear only in the attractive regime, where wide roots can be added freely to the NLIE since their number cancels from the counting equation (6). To describe how these string like structures appear form Eq.(15) note that most of the imaginary roots of the string are in the second determination thus Eq.(15) takes the form

$$
Z(\theta)=2 M L \sinh \theta+P_{\mathrm{bdry}}(\theta)-\sum_{j=n}^{-m}\left(\chi_{I I}^{-}\left(\theta-\theta_{j}\right)+\chi_{I I}^{+}\left(\theta+\theta_{j}\right)\right)
$$

The location of the roots are $\theta_{j}=i \nu_{j}+i \epsilon_{j}(L)$, where we suppose that $\epsilon_{j}(L) \rightarrow 0$ as $L \rightarrow \infty$. If $\Im m\left(\theta_{n}\right)<p \pi$ then the term corresponding to $j=n$ in the sum is replaced with $\chi\left(\theta-\theta_{n}\right)+\chi\left(\theta+\theta_{n}\right)$. The other terms are defined as $f_{I I}^{ \pm}(\theta)=f(\theta)-f(\theta \mp i \pi p)$. We also suppose that $\Im m\left(\theta_{-m}\right)<\pi$ since we need a BBS state with non-vanishing energy.

The position of the roots is determined by the quantization condition $e^{i Z_{I I}^{+}\left(\theta_{k}\right)}=1$ for wide roots and $e^{i Z\left(\theta_{n}\right)}=1$ for the close root if there is one. The condition can be written as

$$
e^{2 i M L \sinh _{I I}^{+}\left(\theta_{k}\right)} \frac{e^{i P_{\mathrm{bdry} \mathrm{II}}^{+}\left(\theta_{k}\right)}}{a\left(2 \theta_{k}\right)} \frac{a\left(2 \theta_{k}-i \pi p\right)^{2}}{a\left(2 \theta_{k}-2 i \pi p\right)} \prod_{j \neq k} \frac{1}{a_{I I}^{+}\left(\theta_{k}+\theta_{j}\right)_{I I}^{+} a_{I I}^{-}\left(\theta_{k}-\theta_{j}\right)_{I I}^{+}}=1
$$

Since $e^{-2 M L \sin \nu_{k}} \rightarrow 0$ in the limit $L \rightarrow \infty$ we have to analyze the singularity structure of the function appearing in Eq.(201). The analysis of the zero string case showed that $\frac{e^{i P_{\text {bry II }}^{+}\left(\theta_{k}\right)}}{a\left(2 \theta_{k}\right)}$ has a pole at $\theta=i \nu_{0}$. Furthermore

$$
a_{I I}^{-}(\theta)_{I I}^{+}=\frac{a^{2}(\theta)}{a(\theta+i \pi p) a(\theta-i \pi p)}
$$


has a pole at $\theta=i \pi p$ and a zero at $\theta=-i \pi p$. Focusing on the divergent terms in Eq.(20) one obtains the equations:

$$
\begin{gathered}
e^{\left(\lambda_{n+1}-\lambda_{n}\right) L} \propto \epsilon_{n}-\epsilon_{n-1} ; \quad e^{\left(\lambda_{n}-\lambda_{n-1}\right) L} \propto \frac{\epsilon_{n-1}-\epsilon_{n-2}}{\epsilon_{n}-\epsilon_{n-1}} ; \quad \ldots ; \quad e^{\left(\lambda_{k}-\lambda_{k-1}\right) L} \propto \frac{\epsilon_{k-1}-\epsilon_{k-2}}{\epsilon_{k}-\epsilon_{k-1}} ; \quad \ldots \\
e^{\left(\lambda_{2}-\lambda_{1}\right) L} \propto \frac{\epsilon_{1}-\epsilon_{0}}{\epsilon_{2}-\epsilon_{1}} ; \quad e^{\left(\lambda_{1}-\lambda_{0}\right) L} \propto \epsilon_{0} \frac{\epsilon_{0}-\epsilon_{-1}}{\epsilon_{1}-\epsilon_{0}} ; \quad e^{\left(\lambda_{0}-\lambda_{-1}\right) L} \propto \frac{\epsilon_{-1}-\epsilon_{-2}}{\epsilon_{0}-\epsilon_{-1}} \ldots ; e^{\left(\lambda_{-m+1}-\lambda_{-m}\right) L} \propto \frac{1}{\left(\epsilon_{-m+1}-\epsilon_{-m}\right)}
\end{gathered}
$$

where $\lambda_{k}=2 M \sin \nu_{k}$ and whenever $\theta_{n}$ is a close root then in the first equation we have to put $\lambda_{n+1}=0$.

Since all the $\epsilon$-s have to go to zero as $L \rightarrow \infty$ these equations lead to the following requirements:

$$
\begin{aligned}
\lambda_{n+1}<\lambda_{n} \quad ; \quad & \lambda_{n+1}<\lambda_{n-1} \quad ; \quad \ldots ; \quad \lambda_{n+1}<\lambda_{1} \\
& \lambda_{-m}>\lambda_{n+1} \\
\lambda_{-m}<\lambda_{-m+1} \quad ; \quad & \lambda_{-m}<\lambda_{-m+2} \quad ; \quad \ldots \quad ; \quad \lambda_{-m}<\lambda_{0}
\end{aligned}
$$

The strongest condition from the first line is $\lambda_{n+1}<\lambda_{1}$ which means $\nu_{1}-\frac{\pi}{2}<\frac{\pi}{2}-\nu_{n+1}$. The second requirement can be translated to $\nu_{-m}-\frac{\pi}{2}<\frac{\pi}{2}-\nu_{n+1}$ which is equivalent to $\nu_{n+1}<\pi-\nu_{-m}=w_{m}$. Finally the strongest condition from the last line is $\lambda_{-m}<\lambda_{0}$ which is equivalent to $w_{m}<\nu_{0}$. These last two conditions are completely equivalent to the ones Saleur-Skorik obtained for the $(n, m)$ strings from the BAE on the one hand, while they are also consistent with the DM bounds (10) on the other.

\subsection{Bound-state NLIE in the repulsive regime}

In this subsection the interpretation of the BBS in the repulsive regime is elaborated. The validity range of the pure NLIE - Eq.(4) without source terms - as it is derived from the BAE using Fourier transformation, is $-2 p-2<H_{ \pm}<2 p+2$. We give its interpretation in this full range. The symmetry $H_{ \pm} \rightarrow H_{ \pm}+2 p+2$ of the BAE survives at the NLIE level (can be checked also by explicit comparison), thus the $[0,2 p+2]$ domain is equivalent to the $[-2 p-2,0]$ domain. One possibility to compare with the DM spectrum is to put a particle between the two boundaries and analyze its reflection factors by comparing the large volume limit of the NLIE with the Bethe-Yang quantization condition. We recall this analysis from [8] here.

In the repulsive regime in the half line theory we have at most one BBS and its energy can be plotted as function of the boundary parameter $\xi$ as shown on Figure 2.

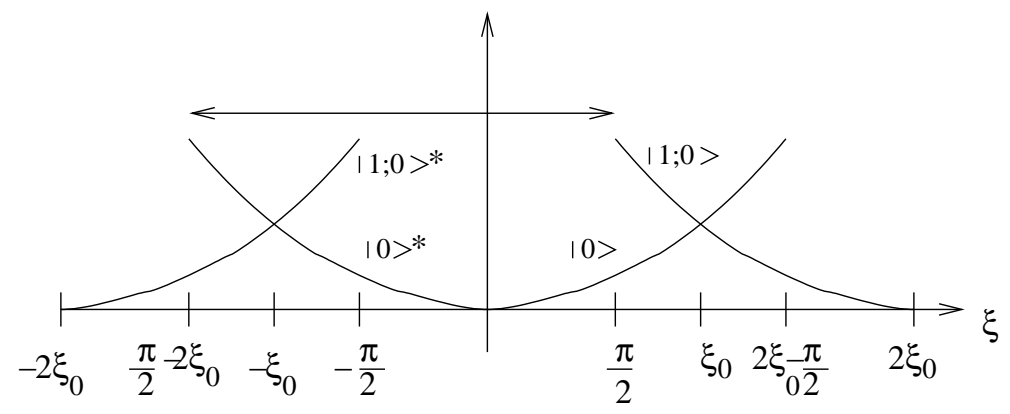

Figure 2: Shematic figure of the boundary condition dependent part of the energies of the various ground-states

Figure 2 is schematic and shows only the $\xi$ dependent part of the boundary energies of the groundstate, $E_{|0\rangle}(\xi)=-\frac{M \cos p \xi}{2 \cos \frac{\pi P}{2}}\left[6\right.$, and the BBS, $E_{|1 ; 0\rangle}=E_{|0\rangle}+\cos \nu_{0}$. The discrete symmetries $\xi \rightarrow-\xi$ and $\xi \rightarrow 2 \xi_{0}-\xi$ induce maps between the states $|0\rangle \rightarrow|0\rangle^{*}$ and $|0\rangle \rightarrow|1 ; 0\rangle$ but in the same time the soliton has to be exchanged with the anti-soliton. The two theories $\xi$ and $-\xi$ are not equivalent so we distinguish their states by star. The transformation $\xi \rightarrow \xi+2 \xi_{0}$ maps $|1 ; 0\rangle^{*} \rightarrow|0\rangle$ and $|0\rangle^{*} \rightarrow|1 ; 0\rangle$ without changing the species. These transformations correspond to the $\phi \rightarrow-\phi, \phi \rightarrow \frac{2 \pi}{\beta}-\phi$ and 
$\phi \rightarrow \phi+\frac{2 \pi}{\beta}$ transformations of the Lagrangian and, by means of them, the parameter range of the theory can be restricted to $\phi \in\left[0, \frac{\pi}{\beta}\right]$ or equivalently to $\xi \in\left[0, \xi_{0}\right]$.

The boundary condition dependent part of the reflection factor of the soliton on the ground-state boundary can be written as (11) which is valid in the domain $0<\xi<\frac{\pi}{2}$. The bound at $\frac{\pi}{2}$ signals the pole of the reflection factor which corresponds to the boundary state $|1 ; 0\rangle$. The integral representation is valid also for $-\frac{\pi}{2}\left(1+2 p^{-1}\right)<\xi<0$ but here it corresponds to the state $|0\rangle^{*}$. The validity range of the integral representation is marked with the arrow on the figure.

Now putting one hole into the pure NLIE for $2 p>H_{ \pm}>0$, and comparing the large volume limit of the quantization condition to the Bethe-Yang equation

$$
e^{i Z\left(\theta_{1}\right)}=1 \quad \longleftrightarrow \quad e^{2 i M L \sinh \theta_{1}} e^{i P_{|0\rangle}\left(\xi_{+}, \theta_{1}\right)} e^{i P_{|0\rangle}\left(\xi_{-}, \theta_{1}\right)}=1
$$

one arrives at the identifications of the parameters (16). Let us fix $H_{-}$in the domain $[2 p, 0]$ such that this boundary does not allow any bound-state and scan the whole $-2 p-2<H_{+}<2 p+2$ range on the other boundary. The previous findings show that the integral equation in the domain $H_{+} \in[0, p]$ describes the ground state $|0\rangle$. They also show that in the range $H_{+} \in[p, 2 p+2]$ it describes the $|0\rangle^{*}$ state instead. This is interesting since for $H_{+} \in[2 p, 2 p+1]$ there exists a BBS in the spectrum but the pure NLIE corresponds to the ground-state. The same NLIE in the $H_{+} \in[2 p+1,2 p+2]$ domain describes the BBS, and using the symmetry $H \rightarrow H+2 p+2$ we can conclude that it corresponds also to the excited boundary state $|1 ; 0\rangle$ in the $H_{+} \in[-1,0]$ range. For $H_{+} \in[-2-p,-1]$ it describes the $|1 ; 0\rangle$ ground-state. This can also be confirmed by comparing the reflection factor of the soliton on the state $|1 ; 0\rangle$ to the result coming from the NLIE for $H_{+}<0$.

In summarizing using the reflection factors of the solitonic states we conclude that the pure NLIE describes the state marked with + for $H_{+}>0$ and the one marked with - for $H_{+}<0$ in Figure 3.

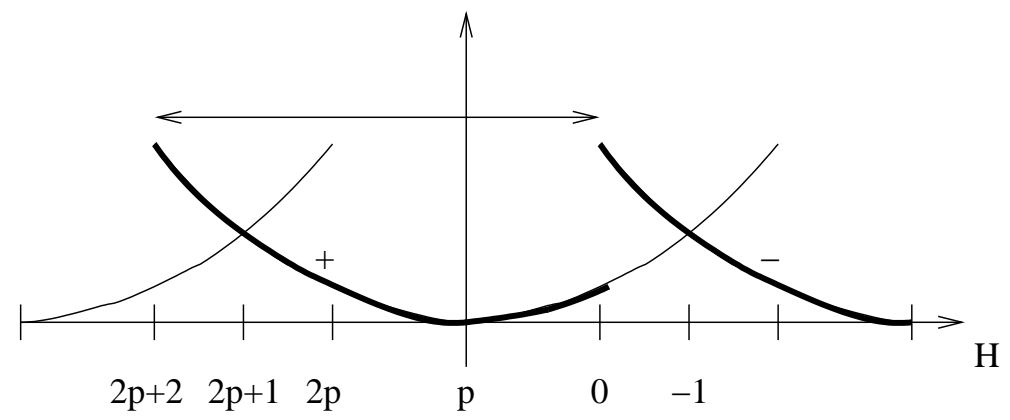

Figure 3: The pure NLIE describes the state marked with +

The boundary energies cannot be calculated either from the NLIE or from the bootstrap since in both approaches the ground-state energy is normalized to 0 and energy differences can be determined only.

Let us introduce a boundary imaginary root in the NLIE (4). The BAE and the asymptotic analysis of the NLIE predict the position of the root to be

$$
\theta=i u \approx i \frac{\pi}{2}\left(n(2 p+2)-H_{+}\right) \quad, \quad n \in Z
$$

which we plot on Figure 4.

On Figure 4 the first determination is below $i \pi$ marked with a dashed line while the self-conjugate line indicated by a dotted line is at $i \frac{\pi}{2}(1+p)$. Exactly when $H_{+}=2 p$ the $n=1$ imaginary root appears at $i \pi$. Adding it to the NLIE via the source term $-\chi(\theta-i u)-\chi(\theta+i u)$ modifies the reflection factor and increases the energy by $-M \cos u$. Clearly this is positive for $H_{+}<2 p+1$ zero for $H_{+}=2 p+1$, negative between $2 p+1<H_{+}<2 p+2$ and leaves the imaginary axis at $H=2 p+2$. So from comparing the energy differences between the pure NLIE and the NLIE with the imaginary root added we suspect that the later one describes the state denoted by the dotted line on Figure 5. 


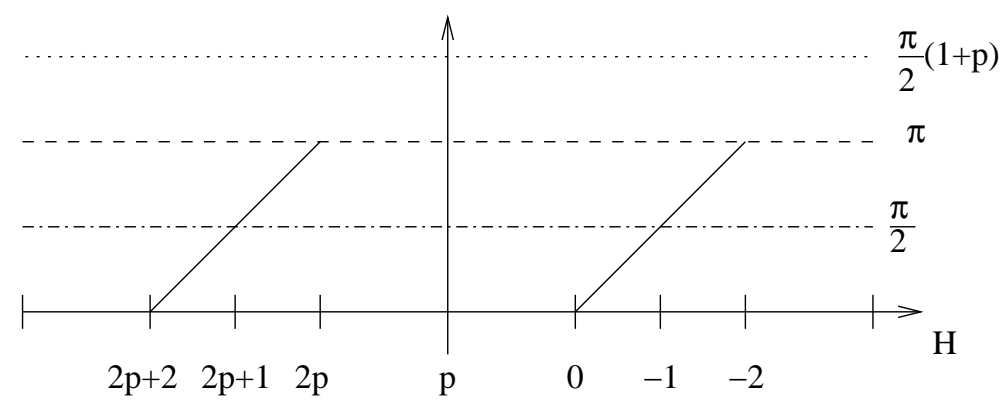

Figure 4: The straight lines show the location of the imaginary root as a function of $H$

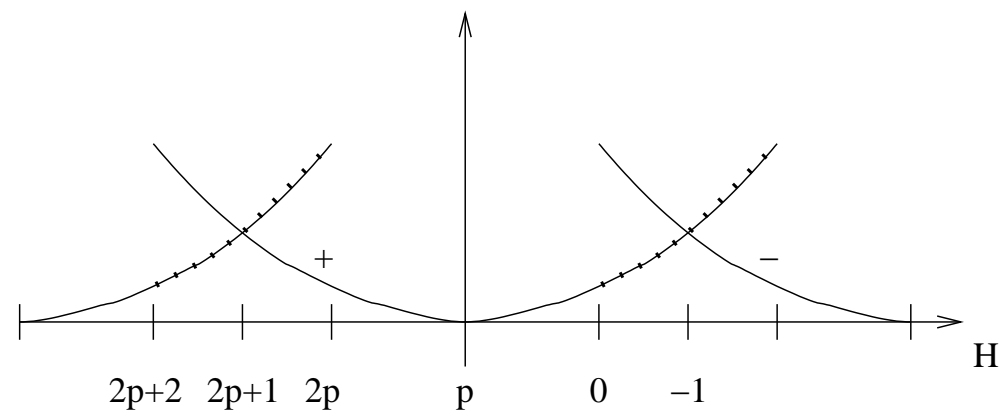

Figure 5: The NLIE with the imaginary root added describes the state marked with the dotted line

This can be confirmed also by putting one additional hole and analyzing the reflection factors, which we show below.

Since all these states can be described in the $p>H_{+}>-1$ or equivalently in the $0<\xi_{+} p<\frac{\pi}{2}(1+p)$ domain we focus on this from now on and summarize the previous findings. The pure NLIE describes the ground-state $|0\rangle$ for the range $p>H_{+}>0$, while for the range $0>H_{+}>-1$ it describes the state $|1 ; 0\rangle$. If we, in this range, add an imaginary root located at $i u_{0}$ (where $u_{0}=\nu_{0}=p \xi-\frac{\pi p}{2}$ ) we change the energy by $-M \cos \nu_{0}$ and the logarithm of the reflection factor by $-\chi\left(u-i \nu_{0}\right)-\chi\left(u+i \nu_{0}\right)$. The energy of this state is then

$$
E=E_{|1 ; 0\rangle}-M \cos \nu_{0}
$$

while the reflection factor is

$$
P^{+}(\theta)=P_{|1 ; 0\rangle}^{+}(\theta) \frac{1}{a\left(\theta-i \nu_{0}\right) a\left(\theta+i \nu_{0}\right)}
$$

from these two expressions we can read off (12) that the state with the imaginary roots at $\pm i \nu_{0}$ added is the ground state $|0\rangle$.

We can argue another independent way that the state with the root added in the $H_{+} \in[0,-1]$ range describes the ground-state. We can perform an analytic continuation of the pure NLIE from the $H_{+}>0$ domain. This method is standard and was used frequently to obtain the excited states in the TBA equations [16, 17, 18. By changing the sign of $H_{+}$two singularities of $\log \left(1-e^{i Z}\right)$ are crossing the contours and by encircling them and picking up the residue terms the NLIE with one root pair added can be obtained from the pure NLIE.

\subsection{Bound-state NLIE in the attractive regime}

In this subsection we map the large volume limit of the boundary strings to the BBSs of DM in the attractive regime. Since all states can be described in the $p>H_{+}>-1$ domain we concentrate only on this range. First we figure out the correspondence from the boundary energies and then confirm our findings by comparing the solitonic reflection factors, too. 
From the bootstrap point of view the properties of the first excited boundary state $|1 ; 0\rangle$, such as energy and reflection factor, are the same as in the repulsive regime and the interpretation of the pure NLIE is completely analogous: for $H_{+}>0$ it describes the ground-state $|0\rangle$, while for $-1<H_{+}<0$ it corresponds to the BBS $|1 ; 0\rangle$. In the attractive regime, however, where breathers are also in the spectrum we can confirm this assignment independently by analyzing the large volume behavior of the first breather.

In doing so we insert a self-conjugate root into the large volume limit of the pure NLIE (44)

$$
Z(\theta)=2 M L \sinh \theta+P_{\mathrm{bdry}}(\theta)-\left(\chi_{I I}(\theta-\alpha)+\chi_{I I}(\theta+\alpha)\right) \quad ; \quad \alpha=\theta_{0}+i \frac{\pi}{2}(p+1)
$$

and compare the $e^{i Z(\alpha)_{I I}}=1$ quantization condition to the first breather's Bethe-Yang equation

$$
e^{i 2 m_{1} L \sinh \theta_{0}} R_{H_{+}}^{(1)}\left(\theta_{0}\right) R_{H_{-}}^{(1)}\left(\theta_{0}\right)=1
$$

Here $m_{1}=2 M \sin \frac{\pi p}{2}$ is the mass of the first breather and

$$
R_{H}^{(1)}(\theta)=\frac{(2+p)_{\theta}(1)_{\theta}}{(3+p)_{\theta}}\left[\frac{(p-|H|-1)_{\theta}}{(p-|H|+1)_{\theta}}\right]^{\operatorname{sign}(H)}, \quad(x)_{\theta}=\frac{\sinh \left(\frac{\theta}{2}+i \frac{\pi x}{4}\right)}{\sinh \left(\frac{\theta}{2}-i \frac{\pi x}{4}\right)}
$$

denotes its reflection factor [19]. Using the integral representation of the combination

$$
(x)_{\theta+i \frac{\pi}{2}}(x)_{-\theta+i \frac{\pi}{2}}=\exp \left[\int_{-\infty}^{\infty} \frac{d t}{t} e^{\frac{i t \theta}{\pi}} \frac{\sinh t\left(1-\frac{x}{2}\right)}{\cosh (t / 2)}\right]
$$

together with the identity $(1+p)_{i \frac{\pi}{2} \mp \theta}=(1-p)_{i \frac{\pi}{2} \pm \theta}$ one can indeed map the quantization condition (15) to the Bethe-Yang equation (21). Furthermore, from the first breather's reflection amplitude emerging from this comparison one can see that the pure NLIE describes the ground-state $|0\rangle$ for $H_{+}>0$, while for $H_{+}<0$ it gives the BBS $|1 ; 0\rangle$.

Once we know the interpretation of the pure NLIE we turn to the analysis of the $(n, m)$ string allowed by both the BAE and the asymptotic analysis of the NLIE. We include the following source term in the NLIE

$$
\text { source }=-\sum_{j=-n+1}^{m}\left(\chi_{I I}\left(\theta-i u_{j}\right)+\chi_{I I}\left(\theta+i u_{j}\right)\right)-\left\{\begin{array}{c}
\chi_{I I}\left(\theta-i u_{-n}\right)+\chi_{I I}\left(\theta+i u_{-n}\right) \text { if } u_{-n}>\pi p \\
\chi\left(\theta-i u_{-n}\right)+\chi\left(\theta+i u_{-n}\right) \text { if } u_{-n}<\pi p
\end{array}\right.
$$

and at the same time make the corresponding modification of the energy

$$
E=-M \sum_{j=-n+1}^{m} \cos _{I I}\left(u_{j}\right)-\left\{\begin{array}{ccc}
\cos _{I I}\left(u_{-n}\right) & \text { if } \quad u_{-n}>\pi p \\
\cos \left(u_{-n}\right) & \text { if } \quad u_{-n}<\pi p
\end{array}\right.
$$

The contribution of the wide and close roots reads explicitly

$$
\text { source }=-\chi\left(\theta-i u_{m}\right)-\chi\left(\theta+i u_{m}\right) \quad ; \quad E=-M \cos u_{m}
$$

if $u_{-n}<p \pi$ and

source $=-\chi\left(\theta-i u_{m}\right)-\chi\left(\theta+i u_{m}\right)+\chi\left(\theta-i u_{-n-1}\right)+\chi\left(\theta+i u_{-n-1}\right) \quad ; \quad E=-M \cos u_{m}+M \cos u_{-n-1}$

if $u_{-n}>p \pi$. The NLIE and the BAE is periodic with period $i \pi(1+p)$. From now on we use the imaginary strip between 0 and $\pi(1+p)$ as the fundamental range in contrast to the usual $\left[-\frac{\pi}{2}(p+\right.$ 1), $\frac{\pi}{2}(p+1)$ ]. Roots with negative imaginary parts are mapped to the upper half plane by the $u \rightarrow$ $u+\pi(1+p)$ transformation as is demonstrated on Figure 6 . 


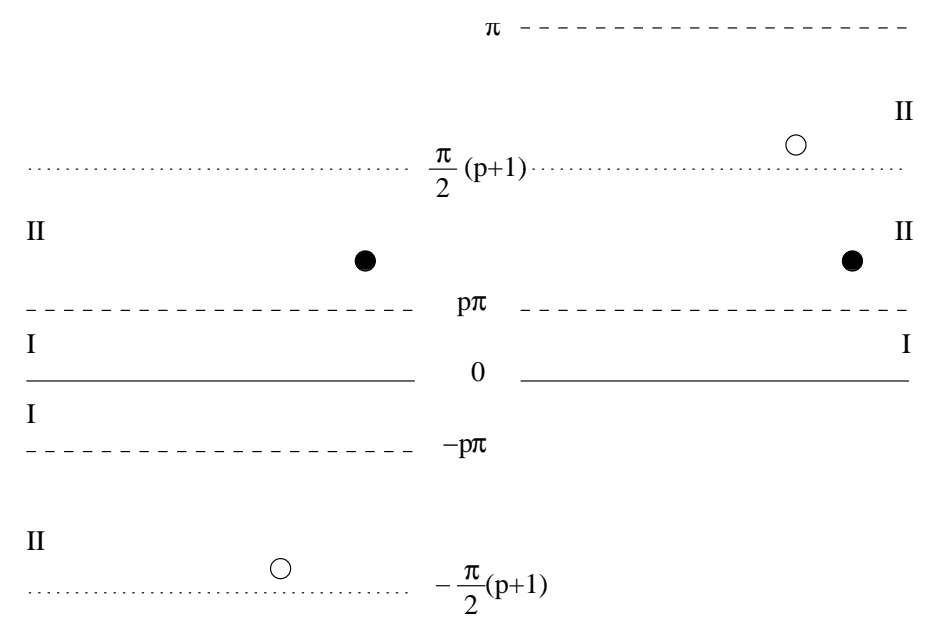

Figure 6: The two fundamental strips used to describe imaginary roots

Taking any allowed root $-\pi p>\bar{u}>-\frac{\pi}{2}(p+1)$ we replace it with the root $u=\bar{u}+\pi(p+1)$ which is now in the strip $\frac{\pi}{2}(p+1)<u<\pi$. In the energy formula the corresponding change is to replace $\cos _{I I}(\bar{u})$ by $\cos _{I I}(u)$, but they are equal since

$$
\cos _{I I}(\bar{u})=\cos (\bar{u})-\cos (\bar{u}+\pi p)=\cos (u-\pi p-\pi)-\cos (u-\pi)=\cos u-\cos (u-\pi p)=\cos _{I I}(u)
$$

Similarly in the source term we replace $\chi_{I I}(\theta-i \bar{u})+\chi_{I I}(\theta+i \bar{u})$ by $\chi_{I I}(\theta-i u)+\chi_{I I}(\theta+i u)$. Their equality follows from the fact that $\chi_{I I}(\theta-i u)+\chi_{I I}(\theta+i u)$ is symmetric for $u=\frac{\pi}{2}(p+1)$, which is a consequence of the identity (13).

Let's analyze now the boundary energy as well as the reflection factors of the solitons in case of the $(n, m)$ string added. To make correspondence with the BAE we remark that $\nu_{0}=u_{0}=\xi p-\frac{\pi p}{2}$. As a consequence

$$
u_{-n}=\nu_{n} \quad ; \quad u_{m}=\pi-w_{m}
$$

We start the analysis with the simplest $m=0$ string. We have to distinguish two cases as shown on Figure 7.

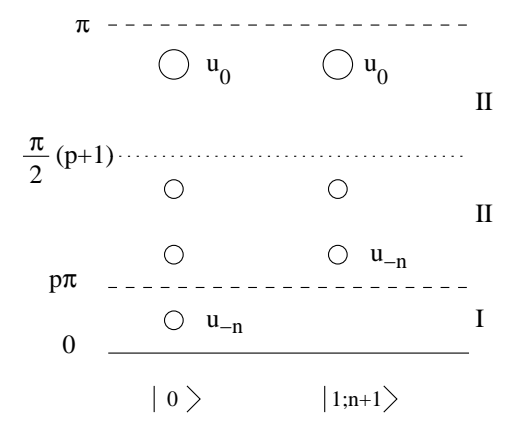

Figure 7: The figure of the two possible $(n, 0)$ strings

In the first case (left) $u_{-n}<\pi p$. The energy compared to the state $|1 ; 0\rangle$ (described by the pure NLIE) is

$$
E=E_{|1 ; 0\rangle}-M \sum_{j=n}^{0} \cos _{I I} \nu_{j}-M \cos \nu_{n}=E_{|1 ; 0\rangle}-M \cos \nu_{0}
$$

while for the reflection factor we obtain the factors

$$
P^{+}(\theta)=P_{|1 ; 0\rangle}^{+} a\left(\theta-i \nu_{0}\right)^{-1} a\left(\theta+i \nu_{0}\right)^{-1}
$$


This is the same result we obtained in the repulsive regime when added one root at $i \nu_{0}$ thus we conclude that it describes the ground-state $|0\rangle$. So the ground-state corresponds to the longest string and this is true as far as $0<\nu_{0}<\frac{\pi}{2}$ which is equivalent to $0>H_{+}>-1$. This result was obtained also by [11]. Let us note that we can describe the ground-state by analytic continuation in $H_{+}$as we did in the repulsive case. The difference being, that once $H_{+}$reaches the value when $\nu_{n}\left(H_{+}\right)$enters the physical strip we have to move it through the integration contour which results in its source term. That is why the longest possible $(0, n)$ string gives the vacuum.

Suppose now that $u_{-n}>\pi p$ (right on the figure) so the bottom root is in the second determination, too. Using the second determination of the cosine function we obtain the energy as

$$
E=E_{|1 ; 0\rangle}-\cos \nu_{0}+\cos \nu_{n+1}
$$

while the reflection factor turns out to be

$$
P^{+}(\theta)=P_{|1 ; 0\rangle}^{+} \frac{a\left(\theta-i \nu_{n+1}\right) a\left(\theta+i \nu_{n+1}\right)}{a\left(\theta-i \nu_{0}\right) a\left(\theta+i \nu_{0}\right)}
$$

This is all consistent with the proposal that this string corresponds to the state $|1 ; n+1\rangle$. From the DM analysis we know that this state exists when $\nu_{n+1}>0$ which is just the statement $u_{-n}>\pi p$ we obtained from the asymptotic analysis.

Consider now the most general $(n, m)$ string with $m>0$. Distinguish again two cases depending on whether $u_{-n}<\pi p$ or $u_{-n}>\pi p$ as shown on Figure 8.

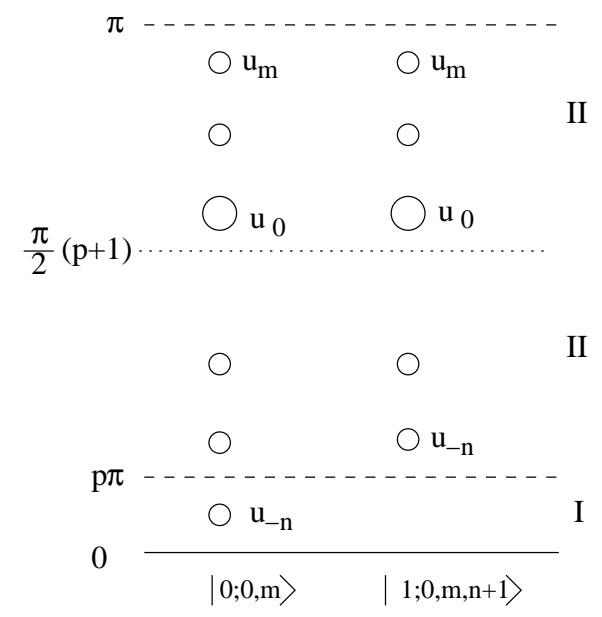

Figure 8: The figure of the two possible $(n, m)$ strings

In the first case (left) $u_{-n}<\pi p$ the energy of an $(n, m)$ string is

$$
E=E_{|1 ; 0\rangle}-\cos u_{m}=E_{|1 ; 0\rangle}+\cos w_{m}
$$

while the reflection factor is

$$
\begin{aligned}
P^{+}(\theta) & =P_{|1 ; 0\rangle}^{+} \frac{1}{a\left(\theta+i\left(\pi-w_{m}\right)\right) a\left(\theta-i\left(\pi-w_{m}\right)\right)} \\
& =P_{|0\rangle}^{+} \frac{a\left(\theta-i \nu_{0}\right) a\left(\theta+i \nu_{0}\right)}{a\left(\theta+i\left(\pi-w_{m}\right)\right) a\left(\theta-i\left(\pi-w_{m}\right)\right)}
\end{aligned}
$$

Both the energy and the reflection factor is consistent with the identification of the string with the $|0 ; 0, m\rangle$ BBS. From the DM bootstrap analysis we know that this bound-state exists whenever $\frac{\pi}{2}>$ $\nu_{0}>w_{m}>0$. The $\nu_{0}>w_{m}$ condition is equivalent to condition $u_{1}+u_{m}>\pi(p+1)$ as obtained from the asymptotic analysis. The $w_{m}>0$ condition is equivalent to $u_{m}<\pi$ which follows from the continuum counting equation (6) 
For $u_{-n}>\pi p$ the energy of the $(n, m)$ string is

$$
E=E_{|1 ; 0\rangle}+\cos w_{m}+\cos \nu_{n+1}
$$

while the reflection factor is

$$
P^{+}(\theta)=P_{|1 ; 0\rangle}^{+} \frac{a\left(\theta+i \nu_{n+1}\right) a\left(\theta-i \nu_{n+1}\right)}{a\left(\theta+i\left(\pi-w_{m}\right)\right) a\left(\theta-i\left(\pi-w_{m}\right)\right)}
$$

From which we can conclude that the corresponding state is $|1 ; 0, m, n+1\rangle$. This state exists whenever $\nu_{0}>w_{m}>\nu_{n+1}>0$. The new condition compared to the previous discussions is $w_{m}>\nu_{n+1}$ but this is equivalent to $u_{-n}+u_{m}<\pi(p+1)$. So this state exists exactly the same time when the corresponding $(n, m)$ string in the BAE.

Suppose now that to the $\left(n_{1}, m_{1}\right)$ string we have already described we add another $\left(n_{2}, 0\right)$ string with $n_{2}<n_{1}$. Since $u_{-n_{2}}>\pi p$ the second string increases the energy by $-M \cos \nu_{0}+M \cos \nu_{n_{2}+1}$ so changes the zero label to $n_{2}+1$. Explicitly if the original state was $\left|0 ; 0, m_{1}\right\rangle$ then the new state is $\left|0 ; n_{2}+1, m_{1}\right\rangle$ if, however, the original was $\left|1 ; 0, m_{1}, n_{1}+1\right\rangle$ then the new state is $\left|1 ; n_{2}+1, m_{1}, n_{1}\right\rangle$. If additionally to the $\left(n_{1}, m_{1}\right)$ string we add another $\left(n_{2}, m_{2}\right)$ string with $n_{2}<n_{1}$ and $m_{2}<m_{1}$, then, since $u_{-n_{2}}>\pi p$, the second string increases the energy by $\cos w_{m_{2}}+\cos \nu_{n_{2}+1}$ so adds two labels. Concretely if the original state was $\left|0 ; 0, m_{1}\right\rangle$ then the new state is $\left|0 ; 0, m_{2}, n_{2}+1, m_{1}\right\rangle$, if, however, it was $\left|1 ; 0, m_{1}, n_{1}+1\right\rangle$ then the new state is $\left|1 ; 0, m_{2}, n_{2}+1, m_{1}, n_{1}+1\right\rangle$. We have checked explicitly that the energy formulas and the reflection factors are consistent with these assumptions. For the existence of this state the bootstrap gives the relation $\nu_{n_{2}+1}>w_{m_{1}}$ but we were not able to find its analogue on the BAE side.

\subsection{Finite size correction of the ground-state energy: boundary Lüscher correc- tion}

In this subsection the large volume asymptotic of the ground-state NLIE is analyzed and compared to the Lüscher type correction [12. The general form of this correction, valid in any two dimensional boundary quantum field theory, was determined in [12] and first we concretize the results for the sine-Gordon model with Dirichlet boundary condition.

In the repulsive regime $(p>1)$, where there is no breather in the spectrum, the finite size energy correction in leading order is governed by the soliton/anti-soliton reflection contribution as

$$
E_{0}(L)=E_{0}(\infty)-M \int_{-\infty}^{\infty} \frac{d \theta}{4 \pi}\left[K_{\alpha}^{-+}(-\theta) K_{\beta}^{+-}(\theta)+K_{\alpha}^{+-}(-\theta) K_{\beta}^{-+}(\theta)\right] e^{-2 M L \cosh \theta}+\ldots
$$

where, the boundary fugacities can be expressed in terms of the soliton/anti-soliton reflection factors: $K_{\alpha}^{+-}(\theta)=R_{+}^{+}\left(i \frac{\pi}{2}-\theta\right)_{\alpha}$ and $K_{\alpha}^{-+}(\theta)=R_{-}^{-}\left(i \frac{\pi}{2}-\theta\right)_{\alpha}$.

If, however, we are in the attractive domain then the leading finite size correction is given by the one particle boundary coupling terms of the breathers:

$$
E_{0}(L)=E_{0}(\infty)-m_{n} \frac{g_{\alpha}^{n} g_{\beta}^{n}}{4} e^{-m_{n} L}+\ldots \quad ; \quad m_{n}=2 M \sin \left(\frac{n p \pi}{2}\right)
$$

If the one particle terms of the lightest particle, (the first breather), are non-vanishing $g_{\alpha}^{1} g_{\beta}^{1} \neq 0$ then the corresponding term provides the leading finite size correction. If any of them is zero (symmetric boundary with $\phi_{0}=0$ ) then the leading finite size correction is determined by the second breather's term since $g_{\alpha}^{2}$ is never vanishing.

We are going to recover this behavior from the ground-state NLIE separately for the attractive and in the repulsive regimes. In the repulsive case the ground state energy can be described either by the pure NLIE for $H_{+}>0$ or by including the source term corresponding to an imaginary root for $H_{+}<0$. Since the analysis was already done in the first case in [12] we focus on the second possibility. The asymptotic form of the NLIE for large volume can be written as 


$$
Z(\theta)=2 M L \sinh \theta+P_{\text {bdry }}(\theta)-\chi\left(\theta-\theta_{0}\right)-\chi\left(\theta+\theta_{0}\right) \quad ; \quad e^{i Z\left(\theta_{0}\right)}=1
$$

where we neglected the exponentially small corrections coming from the convolution term. We plug this expression into the energy formula

$$
E=-M \cosh \theta_{0}-M \Im m \int_{-\infty}^{\infty} \frac{d \theta}{2 \pi} \sinh (x+i \eta) \log \left(1-e^{i Z(\theta+i \eta)}\right)
$$

and shift the integration contour to $\eta=\frac{\pi}{2}$. In doing so we need to know the analytic structure of $P_{\mathrm{bdry}}(\theta)$. Using the relation coming from the soliton quantization condition [8] we can rewrite the boundary fugacity in terms of the soliton reflection factors on the two boundaries and the solitonsoliton scattering as in (17). This provides the analytic continuation into the domain where the original integral representation is not valid. For $H_{+}<0$ the appearing (excited state) reflection factor has a pole at $i \nu_{0}$. This pole is exponentially closely accompanied with a logarithm of zero singularity at $\theta_{0}$. In shifting the contour we take care of these singularities by encircling them with the contour. We use that $\oint \frac{d \theta}{2 \pi} \frac{d g(\theta)}{d \theta} \log (f(\theta))= \pm i g\left(i u_{ \pm}\right)$, (where \pm applies whenever at $u_{ \pm}$the function $f$ has a pole/zero) and obtain the contribution of the singular terms:

$$
-M \cos \nu_{0}+M \cosh \theta_{0}
$$

The volume $\left(\theta_{0}\right)$ dependent terms cancel, the term $-M \cos \nu_{0}$ gives contribution to the boundary energy $\left(E_{0}(\infty)\right)$ while the integral term with its contour shifted to $i \frac{\pi}{2}$ gives the same integral term it gave in the $H_{+}>0$ case and reproduces the expected correction. The cancellation of the volume dependent terms is the consequence of the fact, that the ground state NLIE with the source term $\left(H_{+}<0\right)$ can considered as the analytic continuation of the ground state (and pure) NLIE from the $H_{+}>0$ domain in $H_{+}$.

In the attractive regime things are more complicated even for the $H_{+}>0$ case, where there is now BBS in the spectrum. Even if $e^{i P_{\mathrm{bdry}}(\theta)}$ does not contain boundary dependent poles in the physical strip it has poles which correspond to boundary Coleman-Thun mechanisms [20]. Both the reflection factors and the bulk scattering matrix contain Coleman-Thun type poles at $u_{+}^{n}=i \pi n p / 2$ corresponding to on-shell diagrams presented on Figure 9.
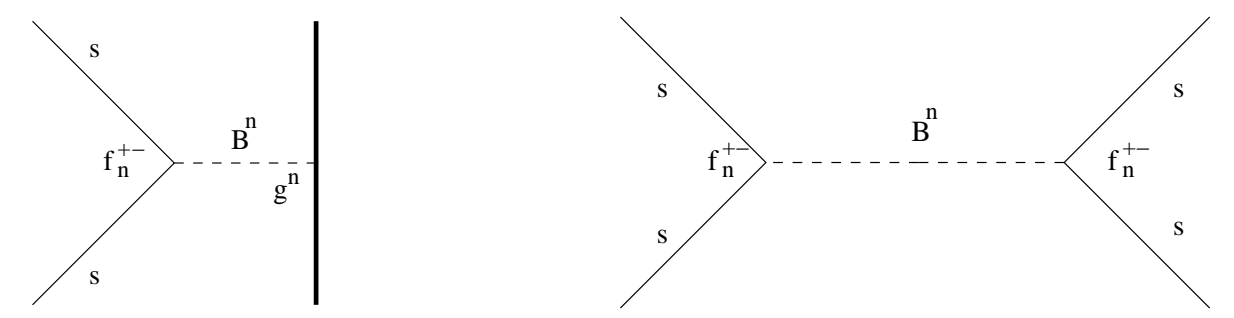

Figure 9: Coleman-Thun diagrams for the reflection and scattering matrices

Since the residues are

$$
R_{+}^{+}(\theta)_{\alpha} \propto-\frac{i}{2} \frac{f_{n}^{+-} g_{\alpha}^{n}}{\theta-i u_{+}^{n}} \quad ; \quad a(2 \theta) \propto-i \frac{f_{n}^{+-} f_{n}^{+-}}{2 \theta-2 i u_{+}^{n}}
$$

we conclude that $e^{i P_{\mathrm{bdry}}(\theta)}$ has single poles at $\theta=i u_{+}^{n}$ with residues

$$
e^{i P_{\mathrm{bdry}}(\theta)} \propto \frac{i}{2} \frac{g_{\alpha}^{n} g_{\beta}^{n}}{\theta-i u_{+}^{n}}
$$

In the exponentially small neighborhood of these poles there are also logarithm of zero singularities in the energy integral at

$$
\left(1-e^{i P_{\mathrm{bdry}}\left(i u_{-}^{n}\right)+2 i M L \sinh \left(i u_{-}^{n}\right)}\right)=0 \quad ; \quad u_{-}^{n} \approx u_{+}^{n}+\frac{g_{\alpha}^{n} g_{\beta}^{n}}{2} e^{-m_{n} L}
$$


We calculate the contributions from the poles and zeros as before and obtain the terms

$$
-M \sum_{n}\left[\cos \left(\theta+i u_{+}^{n}\right)-\cos \left(\theta+i u_{-}^{n}\right)\right]=-\sum_{n} m_{n} \frac{g_{\alpha}^{n} g_{\beta}^{n}}{4} e^{-m_{n} L}
$$

which exactly reproduces the breather's one particle contributions in the range $H_{+}>0$, since the contribution of the shifted integral is of order $e^{-2 M L}$.

In the $H_{+}<0$ domain we have to include the longest allowed boundary string to describe the ground state and additionally to take into account the boundary dependent singularities of (1 $\left.e^{i P_{\mathrm{bdry}}(\theta)+2 i M L \sinh (\theta)}\right)$ when we shift the contour. Since the NLIE with the source terms can be considered as the analytic continuation of the pure NLIE from the $H_{+}>0$ domain we can see that the terms coming from the accompanying zeros of the boundary dependent singularities of $e^{i P_{\mathrm{bdry}}}$ cancel with the volume dependent string energies as it was the case in the repulsive regime.

\section{UV Behavior}

In this section we compute the ultraviolet (UV) behavior of the various energy levels.

As $l:=M L \rightarrow 0$, one can calculate the Casimir energy analytically by using the asymptotic solution of the NLIE (4). From this it is possible to extract the effective central charge defined by

$$
c_{\mathrm{eff}}(l)=-\frac{24 L}{\pi} E(L)
$$

where $E(L)$ is given by Eq.(7). In the UV limit, one can show that only roots and holes growing as $-\log l$ can contribute to $c_{\text {eff }}$. Rescaling the roots and rapidities as

$$
\theta \rightarrow \theta-\log l
$$

and introducing the kink counting function $Z_{+}(\theta)=Z(\theta-\log l)$ together with

$$
Q_{+}(\theta)=2 \Im m \log \left(1-e^{i Z_{+}(\theta)}\right)
$$

one can express the effective central charge as

$$
c_{\mathrm{eff}}(0)=\frac{12}{\pi}\left[-\sum_{k} c_{k} e^{\theta_{k}}+\int_{-\infty}^{\infty} \frac{d \theta}{2 \pi} e^{\theta} Q_{+}(\theta)\right] .
$$

In addition, the NLIE can be rewritten for the kink counting function $Z_{+}(\theta)$ as

$$
Z_{+}(\theta)=e^{\theta}+g_{+}\left(\theta \mid\left\{\theta_{k}\right\}\right)+\sigma-2 i \operatorname{Im} \int d x G(\theta-x-i \epsilon) \log \left[1-e^{i Z_{+}(x+i \epsilon)}\right],
$$

where

$$
g_{+}\left(\theta \mid\left\{\theta_{k}\right\}\right)=\sum_{k} c_{k} \chi_{(k)}\left(\theta-\theta_{k}\right)
$$

and

$$
\sigma=P_{\text {bdry }}(\infty)+2\left(2 S^{0}+S^{+}\right) \chi(\infty) .
$$

Here we defined two integers by

$$
S^{a}=N_{H}^{a}-2 N_{S}^{a}-M_{C}^{a}-2 M_{W}^{a} \operatorname{step}(p-1), \quad a=0,+,
$$

where $N_{H}^{0}$ is the number of holes which do not grow in the $l \rightarrow 0$ limit, etc. 
By following the standard NLIE method [6], one can derive an expression for $\Delta_{0}$, defined by $c_{\text {eff }}=1-24 \Delta_{0}$. It is given by

$$
\Delta_{0}=\frac{1}{8 \pi^{2}} \frac{2 p}{p+1}\left[P_{\mathrm{bdry}}(\infty)+\pi+2 \pi\left(K+S^{0}\right)+2 \pi \frac{p+1}{2 p} S\right]^{2} .
$$

Here the integer $K$ is introduced to relate $Q_{+}(-\infty)$ to $Z_{+}(-\infty)$ by

$$
Q_{+}(-\infty)=Z_{+}(-\infty)+\pi+2 \pi K,
$$

using the definition of $Q_{+}$in Eq.(22). Also $S \equiv S^{0}+S^{+}$can be expressed from Eq.(23) by

$$
S=N_{H}-2 N_{S}-M_{C}-2 M_{W} \operatorname{step}(p-1) .
$$

Since $Q_{+}(-\infty)$ should be given in the fundamental domain of the log function, the integer $K$ should be fixed in such a way that

$$
-\pi<Q_{+}(-\infty) \leq \pi .
$$

One can relate (24) to that of the $c=1$ conformal field theory with Dirichlet boundary condition with compactifying radius $R$ given by

$$
R=\frac{\sqrt{4 \pi}}{\beta}=\sqrt{\frac{p+1}{2 p}} .
$$

which describes the UV limit of the Dirichlet sine-Gordon model. One can easily calculate the boundary term from Eq.(5)

$$
P_{\mathrm{bdry}}(\infty)=\pi+\pi \frac{2 p}{p+1}\left[\operatorname{sign}\left(H_{+}\right)+\operatorname{sign}\left(H_{-}\right)-\frac{H_{+}+H_{-}+2}{p+1}\right] .
$$

Using this and Eq.(8)

$$
\Delta_{0}=\frac{1}{2}\left[\frac{\phi_{+}-\phi_{-}}{\sqrt{\pi}}+m R+\frac{1}{R}\left(K+S^{0}+1\right)\right]^{2},
$$

where the winding number $m$ is defined by

$$
m=\frac{1}{2}\left(\operatorname{sign}\left(H_{+}\right)+\operatorname{sign}\left(H_{+}\right)\right)-1-S .
$$

Writing here $S$ in terms of the holes and imaginary roots as in Eq.(25) one can see that this is the continuum counting equation introduced in Eq.(6).

For the Dirichlet boundary condition, the momentum mode (i.e. the term proportional to $1 / R$ ) in the conformal dimension (27) must vanish. This gives a condition

$$
K+S^{0}+1=0
$$

which fixes the integer $K$. If this condition is met, Eq.(26) can be written as

$$
\delta-\frac{3}{2}+\frac{p}{p+1}<S^{0}<\delta-\frac{1}{2}+\frac{p}{p+1}
$$

where $\delta$ defined by

$$
\delta=\frac{s_{+}+s_{-}}{2}-\frac{\gamma}{2 \pi}\left(H_{+}+H_{-}\right)
$$

takes values in the domain $-1<\delta<1$. With this bound, Eq.(28) restricts possible values of $S^{0}$ strongly. For the repulsive case $p>1$, the allowed values are $S^{0}=-1,0,1$ while they are $S^{0}=-2,-1,0$ for the attractive case $p<1$. As a special example, let us consider a case where only imaginary roots exist. Since these roots can not have large real parts in the UV limit, the number of these roots should be identified with $-S^{0}$. This means a possible number of imaginary roots in the repulsive case is either 0 or 1 , which is consistent with the IR analysis in sect. 3 . 


\section{Conclusions}

In this paper we investigated the NLIE involving purely imaginary roots for the DSG model on a finite interval $L$. We were interested in describing the DM BBSs thus we investigated the case when the boundary parameters at one end of $L$ were "trivial" (i.e. excluded the existence of DM type bound states) but on the other end admitted such a state. We found an exact match between the set of DM type bound states and bound state solutions of the NLIE albeit sometimes the correspondence was surprising: it turned out that in certain parameter domains the pure NLIE (i.e. the one without imaginary roots) describes an excited state and one has to add certain appropriate root(s) to get the ground state. We established the equivalence by studying the large $L$ solutions of the NLIE from which we extracted not only the energies but also the reflection factors. In this process we exploited heavily the fact that sometimes the correct NLIE depends on the second determination of certain quantities. We confirmed our findings by calculating the boundary Lüscher corrections for the ground states and by demonstrating that the UV limit of our NLIE reproduces correctly the conformal dimensions of the expected $c=1$ BCFT.

With these achievements in hand one can certainly think of the following problems for future research: first the numerical investigation of these NLIEs to get information about the finite volume behavior of the bound states that asymptotically correspond to DM. Second the extension of the NLIE to the case of two non trivial boundary conditions at the ends of the interval $L$ may also prove interesting: in this case one expects that, for large $L$ at least, certain pairs of DM bound states appear in the spectrum. Recently, using semi-classical quantization for the DSG model, an interesting restriction ('matching rule') was derived for the allowed pairs in 21]. The semi-classical procedure in the theory with the more general perturbed Neumann type boundary condition revealed the existence of some critical volumes $L_{\text {crit }}$ beyond which the bound states ceased to exist. It would be interesting to see whether these statements are valid in the full quantum theory, i.e. whether they are valid for the solutions of the new NLIE. The first step in this direction is to generalize the present discussion to the case when a constraint is satisfied between the two boundary conditions allowing a BAE type solution of the model [22, 23, 24]. The ground-state NLIE in this case was formulated in [7] while the hole excited states were analyzed in [9]. Thus there is an evident need for proceeding with the description of the BBS which shows the same pattern as the Dirichlet one, see 25] for closing the boundary bootstrap in this case.

The boundary sine-Gordon theory is not the only one exhibiting a complex pattern of boundary excited states. Its supersymmetric generalization has an even more complex BBS spectrum [26] and their description based on the generalization of the ground-state NLIE derived in [27] is also an interesting problem.

\section{Acknowledgments}

The authors would like to thank R. Nepomechie and G. Takács for the illuminating discussions and for taking part in the early stages of this work. This research was partially supported by the Hungarian research funds OTKA K60040 and by a cooperation between the Hungarian Academy of Sciences and the Korean KOSEF. CA was supported in part by a Korea Research Foundation Grant funded by the Korean government (MOEHRD) (KRF-2006-312-C00096) and ZB was supported by a Bolyai Scholarship and the EC network "Superstring". FR thanks partial financial support from the INFN Grant TO12, from the Italian Ministry of University and Research through a PRIN fund and from the NATO Collaborative Linkage Grant PST.CLG.980424.

\section{References}

[1] S. Ghoshal and A. B. Zamolodchikov, Int. J. Mod. Phys. A9, 3841 (Erratum-ibid. A9, 4353) (1994) [hep-th/9306002]. 
[2] C. Destri and H. de Vega, Phys. Rev. Lett. 69, 2313 (1992) [hep-th/9203064];

C. Destri and H. de Vega, Nucl. Phys. B438, 413 (1995) [hep-th/9407117].

[3] A. Klümper, M. T. Batchelor and P. A. Pearce, J. Phys. A24, 3111 (1991).

[4] D. Fioravanti, A. Mariottini, E. Quattrini and F. Ravanini, Phys. Lett. B390, 243 (1997) [hep-th/9608091].

[5] C. Destri and H. de Vega, Nucl. Phys. B504, 621 (1997) [hep-th/9701107].

[6] A. LeClair, G. Mussardo, H. Saleur and S. Skorik, Nucl. Phys. B453, 581 (1995) [hep-th/9503227].

[7] C. Ahn and R. I. Nepomechie, Nucl.Phys. B676, 637 (2004) [hep-th/0309261].

[8] C. Ahn, M. Bellacosa and F. Ravanini, Phys. Lett. B595, 537 (2004) [hep-th/0312176].

[9] C. Ahn, Z. Bajnok, R. I. Nepomechie, L. Palla and G. Takács, Nucl. Phys. B714, 307 (2005) [hep-th/0501047].

[10] P. Mattsson and P. E. Dorey, J. Phys. A33, 9065 (2000) [hep-th/0008071].

[11] S. Skorik and H. Saleur, J.Phys. A28, 6605 (1995) [hep-th/9502011].

[12] Z. Bajnok, L. Palla and G. Takács, Nucl.Phys. B716, 519 (2005) [hep-th/0412192].

[13] F. C. Alcaraz, M. Barber, M. T. Batchelor, R. J. Baxter and G. R. W. Quispel, J. Phys. A20, $6397(1987)$.

[14] E. K. Sklyanin, J. Phys. A21, 2375 (1988).

[15] A. B. Zamolodchikov and Al. B. Zamolodchikov, Ann. Phys. 120, 253 (1979).

[16] P. E. Dorey, A. Pocklington, R. Tateo and G. M. T. Watts, Nucl.Phys. B525, 641 (1998).

[17] C. Rim, "Boundary massive sine-Gordon model at the free Fermi limit and RG flow of Casimir energy", [hep-th/0405162].

[18] Z. Bajnok, C. Rim and Al. B. Zamolodchikov "Sinh-Gordon Boundary TBA and Boundary Liouville Reflection Amplitude", [arXiv:0710.4789].

[19] S. Ghoshal, Int.J.Mod.Phys. A9, 4891 (1994).

[20] Z. Bajnok, G. Böhm and G. Takács, Nucl.Phys. B682, 585 (2004).

[21] Z. Bajnok, L. Palla and G. Takács, Nucl.Phys. B702, 448 (2004).

[22] R. I. Nepomechie, J.Phys. A37, 433 (2004).

[23] J. Cao, H.-Q. Lin, K.-J. Shi and Y. Wang, Nucl. Phys. B663, 487 (2003).

[24] R. I. Nepomechie and F. Ravanini, J.Phys. A36, 11391 (2003).

[25] Z. Bajnok, L. Palla, G. Takács and G.Zs. Tóth, Nucl.Phys. B622, 548 (2002).

[26] Z. Bajnok, L. Palla and G. Takács, Nucl.Phys. B644, 509 (2002).

[27] C. Ahn, R. I. Nepomechie and J. Suzuki, Nucl. Phys. B767, 250 (2007) [hep-th/0611136]. 